\title{
Runaway electron diagnostics for the COMPASS tokamak using EC emission
}

\author{
Michal Farnik ${ }^{1,2, *}$, Jakub Urban ${ }^{1}$, Jaromir Zajac ${ }^{1}$, Ondrej Bogar ${ }^{1,3}$, Ondrej Ficker ${ }^{1,2}$, Eva Macusova ${ }^{1}$, Jan Mlynar ${ }^{1,2}$, \\ Jaroslav Cerovsky ${ }^{1,2}$, Mikita Varavin ${ }^{1,2}$, Vladimir Weinzettl ${ }^{1}$, and Martin Hron $^{1}$ \\ ${ }^{1}$ Institute of Plasma Physics of the CAS, Za Slovankou 1782/3, 18200 Prague 8, Czech Rep. \\ ${ }^{2}$ Faculty of Nuclear Sciences and Physical Engineering, Czech Technical University, Brehova 7, 11519 Prague, Czech Rep. \\ ${ }^{3}$ Faculty of Mathematics, Physics and Informatics, Comenius University in Bratislava, Mlynska dolina 6280, 84248 Bratislava, Slo- \\ vakia
}

\begin{abstract}
An electron cyclotron emission (ECE) diagnostic of suprathermal electrons was utilised for runaway electron (RE) experiments purposes in the COMPASS tokamak. Our vertical ECE (V-ECE) system consists of a 16-channel heterodyne radiometer and an E-band horn antenna with a 76.5-88 GHz frequency range frontend. Simulations used for the design of the diagnostic showed a possibility of detecting the emission of lowenergy (50-140 keV) runaway electrons. We realized measurements with both extraordinary (X-) and ordinary (O-) mode linear polarizations. The amplitudes of the X-mode and O-mode signals are similar, which can be explained by depolarised reflected radiation. V-ECE measurements in low-density flattop discharges and in discharges with massive gas injections of high- $Z$ elements show correlations with other RE diagnostics. Our results are in the agreement with the principles of the primary runaway generation mechanisms.
\end{abstract}

\section{Introduction}

Electron cyclotron emission (ECE) diagnostic is usually used for the electron temperature $\left(T_{\mathrm{e}}\right)$ profile and fluctuation measurements in tokamak devices. However, the electron cyclotron emission measured vertically along the line of a constant magnetic field can yield information about the electron velocity distribution function and its evolution during a discharge [1]. This could be used for the direct detection of the runaway electron presence during runaway experiments performed in the COMPASS tokamak [2]. Ray-tracing simulations were used to design and interpret a measured signals. This motivated us to install a vertical ECE diagnostic in the COMPASS tokamak.

The article is structured as follows. In the second section the suprathermal ECE is introduced. Afterwards, in the third section, the experimental setup with simulations are presented. Finally, in the fourth section, first experimental results are given. Conclusions are drawn at the end

\section{Suprathermal Electron Cyclotron Emission}

Every electron moving in an external magnetic field emits electromagnetic radiation at the cyclotron frequency. The finiteness of the Larmor radius implies that the electromagnetic field generated by an electron gyrating with the cyclotron frequency is not simply harmonic and higher harmonics $n \omega_{\mathrm{c}}$ emerge. Thanks to the relativistic effects, distribution function and the Doppler effect, the emitted

*e-mail: farnik@ipp.cas.cz frequencies have a wide spectrum. The frequency at which an electron moving with a velocity $\mathbf{v}$ is able to emit or absorb the waves having a wave vector $\mathbf{k}$, through the $n$-th harmonic contribution, is given by

$$
\omega=n \cdot \omega_{\mathrm{ce}}(B, v)+k_{\|} v_{\|}=n \cdot \omega_{\mathrm{ce}}(B) / \gamma+k_{\|} v_{\|} \cdot
$$

The relativistic effects are represented by the Lorenz gamma factor. The second term on the right hand side denotes the longitudinal Doppler effect, the index $\|$ indicates the component parallel to $\mathbf{B}$.

It is possible to restrain the influence of the varying magnetic field in (1) via a vertically positioned antenna (V-ECE). The toroidal magnetic induction value becomes approximately constant along the line of sight. Thus, the resonance condition depends only on the velocity. In such a situation, the emission at a particular frequency $(\omega)$ for a parallel optical index $N_{\|}$and a particular cyclotron harmonic $(n)$ occurs only from the electrons satisfying the resonance condition

$$
\frac{\omega\left(c-N_{\|} v_{\|}\right)}{n \cdot \omega_{\mathrm{ce}} c}=\sqrt{1-\left(\frac{v_{\|}}{c}\right)^{2}-\left(\frac{v_{\perp}}{c}\right)^{2}} .
$$

This condition gives us a relationship between the frequency and the electron energy where only the emission from certain velocity phase space $\left(v_{\|}, v_{\perp}\right)$ fulfils the condition (2). As a result, one can diagnose the density and the velocity anisotropy of the electrons as a function of the energy at the expense of returning to a chord-averaged measurement.

From the solution of the radiation transfer equation we calculate the optical depth $(\tau)$. To secure the vital practical 
requirement, avoiding the detection of multiple reflections from the wall, a high optical depth $(\tau \gg 1)$ is needed. [3]

\section{Experimental Setup}

The ECE/EBW heterodyne radiometer was utilized in the past on COMPASS for the measurement of electron Bernstein mode waves (EBW) [4]. The radiometer consists of a separated front-end and a 16-channel intermediate frequency receiver with the bandwidth 1.5-15 GHz. Each channel has a second mixer stage, a digitally controlled built-in attenuator $0-30.5 \mathrm{~dB}$ in 0.5 steps, a power detector and a DC amplifier, which provides a linear output voltage of $0-10 \mathrm{~V} / \mathrm{DC}-1 \mathrm{MHz}$ and the bandwidth $850 \mathrm{MHz}$.

The system, shown on the left in Figure 1, contains front-ends with the Ka-band $(26.5-40 \mathrm{GHz})$ or the E-band divided into two subbands $(60-74.5 \mathrm{GHz} / 76.5-90 \mathrm{GHz})$. The receiver uses a vertical perpendicular vacuum chamber port. Horn antenna with a teflon lens is placed behind a silicon port window, as it is shown in Figure 2 and it is connected to the radiometer over a $\sim 2.5 \mathrm{~m}$ waveguide. [4][5]

The cutoff frequencies increase with the electron plasma frequency $\omega_{\text {pe }}$, which is dependent on the electron density $\omega_{\text {pe }} \propto \sqrt{n_{\mathrm{e}}}$. It is favourable to choose the frontend frequencies as high as possible to be able to measure in the highest possible plasma densities. The electron cutoff densities were calculated for $B=1.15 \mathrm{~T}$ at the magnetic axis of the COMPASS tokamak. In the case of the E2-band frequencies $(76.5-90 \mathrm{GHz})$, the minimum of the cutoff density was $n_{\mathrm{eX}}=4.21 \cdot 10^{19} \mathrm{~m}^{-3}$ for the X-mode and $n_{\mathrm{eX}}=7.26 \cdot 10^{19} \mathrm{~m}^{-3}$ for the O-mode. If the density value is well below the shown numbers, the measured wave is not limited by the cutoff area anywhere during its propagation through the whole plasma. This condition is fulfilled in the vast majority of the discharges within the runaway electron dedicated campaigns in COMPASS.

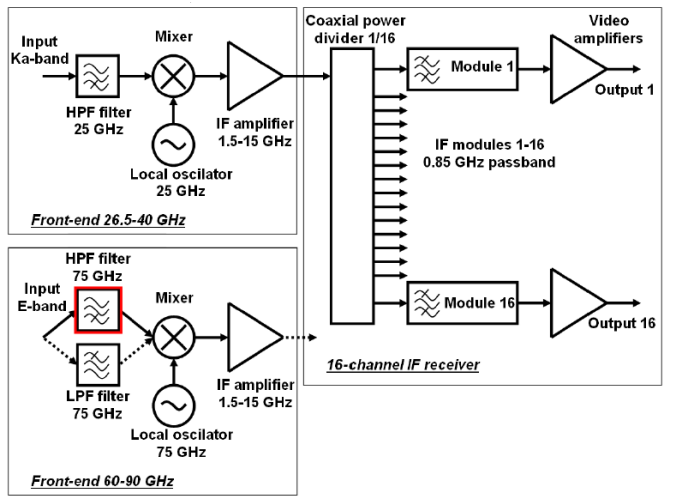

Figure 1. Upper: Block scheme of the ECE/EBW radiometer, taken from [4]

\subsection{Energy Spectrum}

The electron kinetic energies as well as the velocity phase space which fulfil the resonance condition can be determined from the range of frequencies with the use of

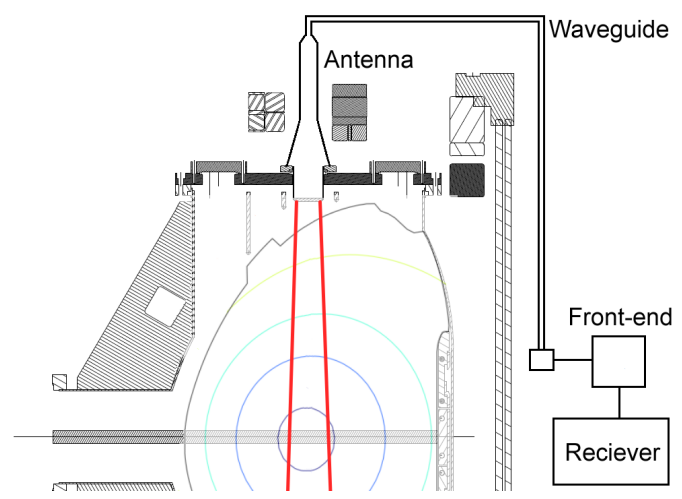

Figure 2. Scheme of the V-ECE measurement setup.

(2). As it was mentioned earlier, the emitted cyclotron frequency is down-shifted in connection with reaching relativistic velocities. Along the line of constant magnetic field $B=1.15 \mathrm{~T}$ the cyclotron frequency has value $v_{\mathrm{ce}}=32.2 \mathrm{GHz}$. This means the E2-band could detect the down-shifted radiation from the third and higher harmonic cyclotron frequency. Figure 3 shows the velocity phase space for the resonance condition and the kinetic energy for the $3^{\text {rd }}$ harmonic frequency of the E2-band.

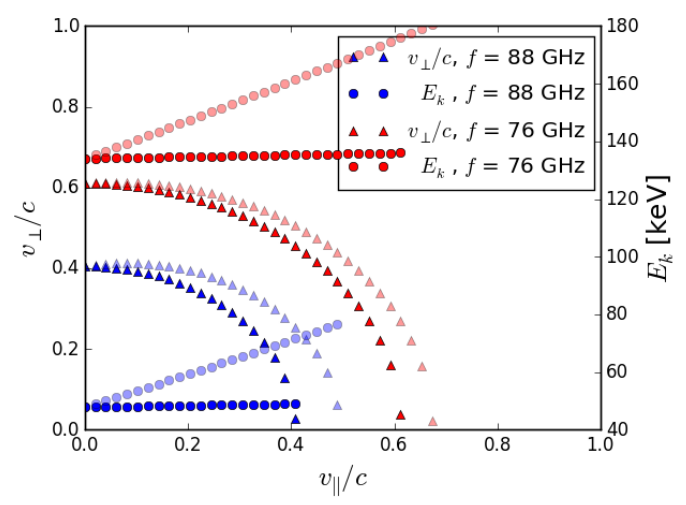

Figure 3. Solution of the resonance condition for the velocities $(\triangle)$ and the electron kinetic energy $E_{\mathrm{k}}(\bullet)$ relevant to the plotted point of the phase space for the $3^{\text {rd }}$ harmonic for the boundary E2-band frequencies. Rich colour: $N_{\|}=0.005$; Pale colour: $N_{\|}=0.1$.

The later given ray-tracing simulations yield the value $N_{\|}=0.005$. Higher $N_{\|}$causes resonance with higher energy electrons. Then, we are able to determine the electron energies in resonance with the E2-band frequencies which are $\sim 50-140 \mathrm{keV}$. Therefore, it could possible to investigate the low-energy REs with the parallel velocities above $0.4 \mathrm{c}$. We can consider the electrons in this phase space "runaway" because of their resonant $v_{\|}$(assuming $v_{\perp}<0.1 \mathrm{c}$ ) is higher than the maximum critical velocity $v_{\mathrm{c}}$ in the V-ECE experiments $\left(n_{\mathrm{e}, \max }=4.2 \cdot 10^{19} \mathrm{~m}^{-3}\right.$, $U_{\text {loop }}=0.7 \mathrm{~V}, \ln \Lambda=13$ ) which is $v_{\mathrm{c}}=0.37 \mathrm{c}$. It corresponds to the kinetic energy $E_{\mathrm{ck}}=36 \mathrm{keV}$. The electrons with velocities higher than the critical velocity are further accelerated by the electric field. They are called the runaway electrons (RE). [6] 

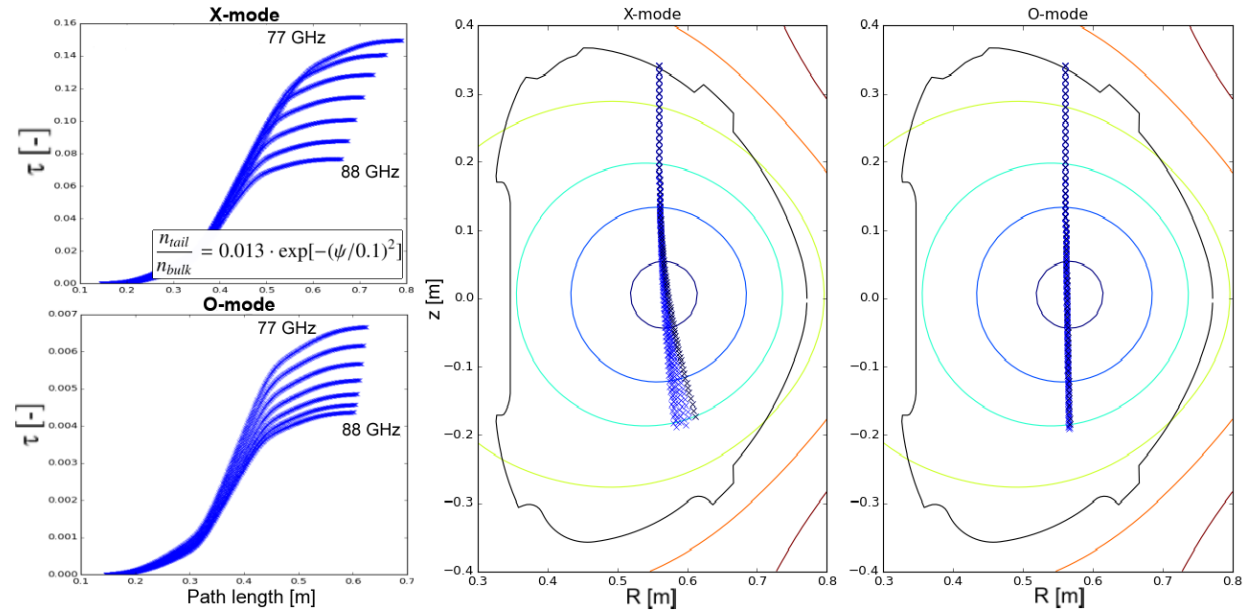

Figure 4. Left: Values of the optical depth $\tau$ dependent on the ray path length $s$ for both O-mode and X-mode for E2-band frequencies. Center and right: Poloidal view of the trajectories of O-mode and X-mode rays in the COMPASS tokamak for odd E2-band frequencies, $B=1.15 \mathrm{~T}$.

\subsection{Ray-tracing Simulations}

The SPECE code [7] was used for the analysis of the electron cyclotron emission from suprathermal electrons. This code solves the radiation transfer equation along the ray trajectories in a tokamak equilibrium. It uses the cold dispersion relation for propagation, while the absorption and emission coefficients are obtained as a solution of the relevant fully relativistic dispersion relation, which is valid at high electron temperatures.

In a Maxwellian plasma, there is a low number of the resonant electrons, therefore neither absorption nor emission occurs in the centre along the line of constant magnetic field $B_{0}$ in the measured frequency range $76.5-88.3 \mathrm{GHz}$. It is necessary to add a non-thermal component into the simulation to increase the absorption coefficient and hence the optical depth. The SPECE can use bi-Maxwellian plasma with added non-thermal component. In our simulations these parameters were used: velocity at the end of the plateau $u_{\max }=0.4 \mathrm{c}$, temperature $T_{\text {tail }}=60 \mathrm{keV}$ distribution function in space and normalised magnetic flux coordinate in Figure 4.

Values of the optical depth for the X-mode are 20 times higher than for the O-mode (see Figure 4). During the evaluation of various simulations this difference between values of the optical depth stayed almost the same. As it was mentioned earlier, the goal is to prevent the detection of waves reflected from the tokamak wall. In the case of $\tau \gg 1$ the reflections are negligible. Unfortunately, the parameters of the plasma and the available radiometer on COMPASS do not provide this suitable condition, which complicates the interpretation of experimental data.

\section{First Experimental Results}

Firstly, it has to be verified that the suprathermal electrons are the source of the measured emission. Due to the possible detection of a reflected emission, the thermal emission could be measured from the $2^{\text {nd }}$ harmonic (HFS) and the $3^{\text {rd }}$ harmonic (LFS) during the ordinary discharges with
$B=1.15 \mathrm{~T}$. In these regions in Figure 5 the non-relativistic electrons fulfil the cold electron cyclotron resonance condition i. e. (1) for $\gamma \rightarrow 1$.

No dependence of the V-ECE signal on the electron temperature has been found in any of the approximately 300 studied discharges. In the discharges with higher magnetic field $B=1.5 \mathrm{~T}$, where the region of the $2^{\text {nd }}$ harmonic thermal emission shifts towards the LFS into the exact centre of the tokamak vessel right in the line of V-ECE diagnostic line of sight. The V-ECE signals in density rampup scenario from the standard and high- $B$ discharge are shown in Figure 6.

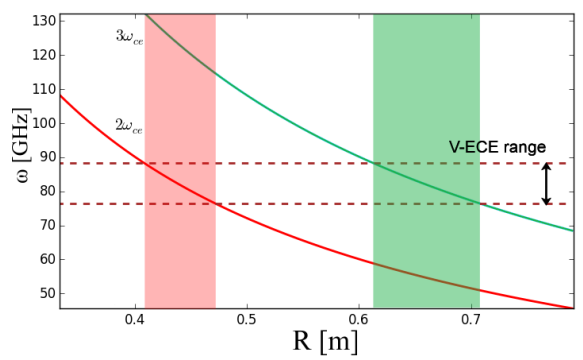

Figure 5. EC frequency profiles for $B=1.15 \mathrm{~T}$ and regions of thermal resonances of the $2^{\text {nd }}$ harmonic (red) and the $3^{\text {rd }}$ harmonic (green) inside the COMPASS plasma.

We do not see any significant differences between V-ECE signals in these two scenarios. Even on the individual channels we do not observe any conspicuous differences. This indicates that the signal comes from the low-energy runaway electrons and does no correlate with electron temperature. The emission intensity from the REs is probably much higher than the thermal emission intensity.

The V-ECE measurements proved to be a good diagnostic of the low-energy runaways and useful tool for detection of the initial runaway seed. A runaway seed significantly influences the evolution of the RE presence within a discharge. These connections are shown in Figure 7. In the yellow rectangle, the difference between a shot with a lower initial RE seed and a shot with a larger initial RE 


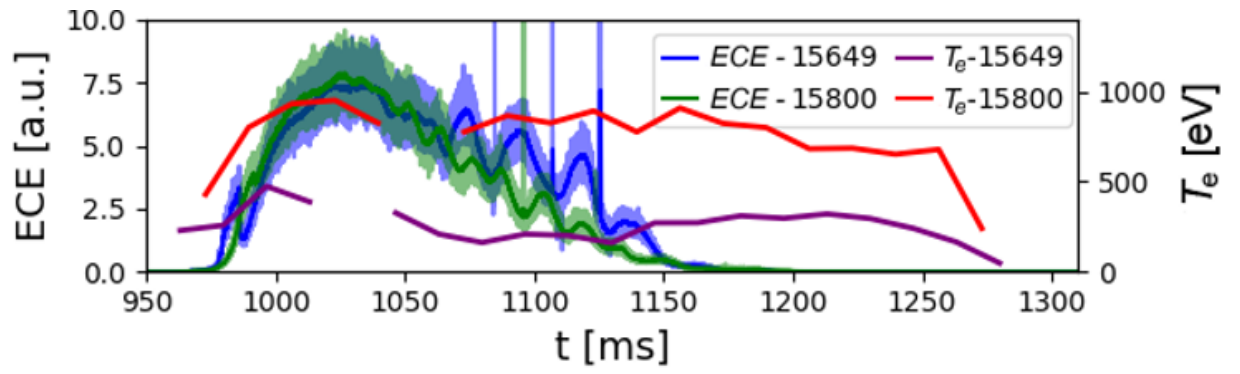

Figure 6. Comparison of the V-ECE measurement and Thomson scattering $T_{\mathrm{e}}$ at the resonance region of the ordinary $B=1.15$ $\mathrm{T}$ (\#15649) and high-field $B=1.50 \mathrm{~T}$ (\#15800) discharge. O-mode, channel $1=76.5 \mathrm{GHz}$ : (O,ch1). Pale colour: raw signal; Rich colour: smoothed signal. $t>1150$ : overdense $\Rightarrow$ cutoff.

seed is highlighted. The primary generation in the shot 14642 is lower due to the higher electron density. Because of the missing whole runaway primary generation, we do not measure strong V-ECE signal neither the signal leap during the discharge. The HXR scintillator measures only the signal during the plasma termination at the end of the discharge (not due to REs). The case of the shot 14643 is the same as it was discussed earlier, it is discharge with the density ramp-down and a large RE population. It is worth mentioning that initial RE losses are not detected by the HXR scintillator. That could imply the confinement of a vast majority of the generated runaways in the initial seed.

By normalising and shifting the HXR signal, we get the confinement time with respect to the initial RE seed and the primary generation. This is shown in Figure 8 . The low-energy runaway population is to some extent terminated as the $24 \mathrm{~ms}$ delayed detection of the HXRs shows us. The REs are accelerated and collide with the tokamak first wall.

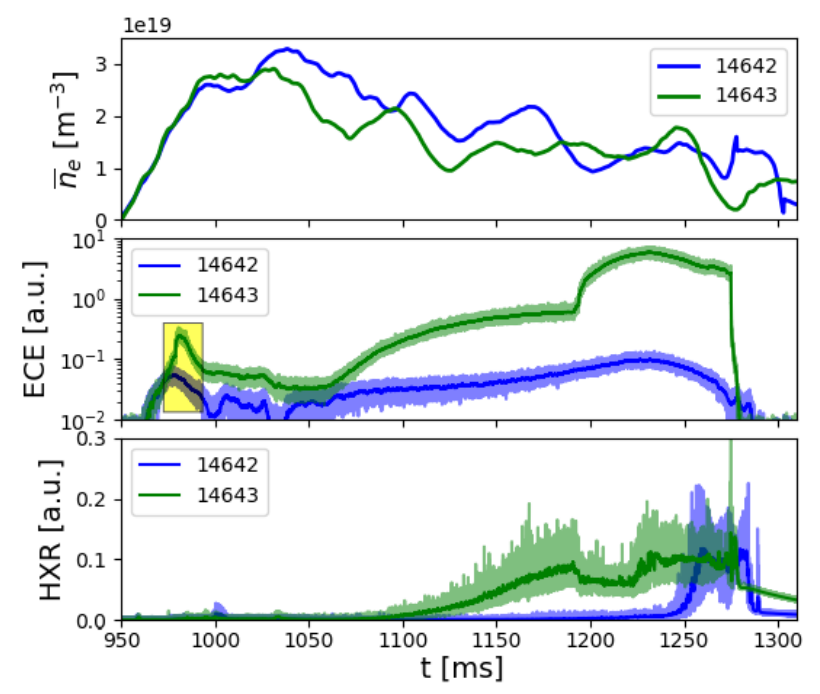

Figure 7. Comparison of the electron density, V-ECE and HXR measurement from the shots 14642 and 14643 . Yellow rectangle highlights the runaway seed. $(\mathrm{O}, \mathrm{ch} 1)$

\section{Conclusion}

The final design of the radiometer was utilized for runaway electron measurements in COMPASS. The V-ECE mea- surement provides information about low-energy runaway electron presence especially about the primary generation at the beginning and in the flat-top phases of the discharge. $\mathrm{V}-\mathrm{ECE}$ can be used as a diagnostic in the RE campaigns.

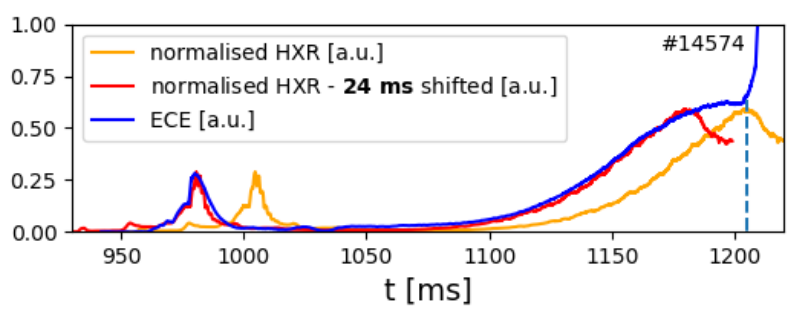

Figure 8. Smoothed HXR and V-ECE signal for the shot 14574. $(\mathrm{X}, \mathrm{ch} 1)$

\section{Acknowledgements}

The author acknowledges Daniela Farina and Lorenzo Figini for providing the SPECE code. This work was supported by the Czech Science Foundation Project No. GA18-02482S.

\section{References}

[1] K. Kato, I.H. Hutchinson, Physical Review Letters 56, 340 (1986)

[2] O. Ficker et al., Nuclear Fusion 57, 076002 (2017)

[3] I.H. Hutchinson, Principles of Plasma Diagnostics (Cambridge University Press, New York, 2002), ISBN 0521803896

[4] J. Zajac, J. Preinhaelter, J. Urban et al., EBE/ECE Radiometry on COMPASS Tokamak-Design and First Measurements, in AIP Conference Proceedings (2009), Vol. 1187, pp. 473-476

[5] J. Zajac, J. Preinhaelter, J. Urban et al., Review of Scientific Instruments 81 (2010)

[6] H. Dreicer, Physical Review 115, 238 (1959)

[7] D. Farina, L. Figini, P. Platania, C. Sozzi, SPECE: A code for electron cyclotron emission in tokamaks, in AIP Conference Proceedings (2008), Vol. 988, pp. 128-131 